\title{
FEMINISMOS EN PODEMOS: CONTRAPODER, DISCURSOS Y ESTRATEGIAS DE LUCHA
}

\author{
Karine Bergès \\ Universidad Paris-Est-Créteil \\ ORCID iD: https://orcid.org/0000-0002-2516-2538 \\ karine_berges@yahoo.fr \\ Eva Espinar-Ruiz \\ Universidad de Alicante \\ ORCID iD: https://orcid.org/0000-0003-2712-4233
}

eva.espinar@ua.es

\section{FEMINISMS IN PODEMOS: COUNTERPOWER, DISCOURSES AND STRATEGIES OF STRUGGLE}

Cómo citar este artículo/Citation: Bergès, K. y Espinar-Ruiz, E. (2020). Feminismos en Podemos: contrapoder, discursos y estrategias de lucha. Arbor, 196 (796): a556. https://doi. org/10.3989/arbor.2020.796n2008

Recibido: 26 mayo 2018. Aceptado: 23 octubre 2019.

RESUMEN: El resurgir en los últimos años de los movimientos feministas en todo el mundo ha llevado a numerosas autoras a hablar de una cuarta ola del feminismo. Este es también el caso de España, donde ha tomado fuerza no solo el movimiento feminista autónomo sino también la participación de grupos y activistas feministas en diferentes protestas, movimientos sociales e incluso partidos políticos. Ejemplo de ello fue la alta implicación de activistas feministas en el movimiento 15-M, muchas de las cuales dieron posteriormente el paso a la representación política, entrando a formar parte de partidos de nueva creación como Podemos. Esta participación constituye una muestra de su compromiso por el cambio social y del reconocimiento de la interseccionalidad de opresiones, pero también exige un complejo ejercicio de reconstrucción y defensa del espacio feminista. Precisamente, en este artículo proponemos el análisis de los debates en torno a propuestas y estrategias que han mantenido las feministas de Podemos que, después de haber militado en movimientos sociales, han decidido colaborar con esta plataforma política.

PALABRAS ClAVE: Feminismos; partidos políticos; Podemos; participación política; compromiso; mujeres en política.
Copyright: ( 2020 CSIC. Este es un artículo de acceso abierto distribuido bajo los términos de la licencia de uso y distribución Creative Commons Reconocimiento 4.0 Internacional (CC BY 4.0).
ABSTRACT: The recent resurgence of feminist movements around the world has led numerous authors to talk about a fourth wave of feminism. This is also the case in Spain, where not only has the autonomous feminist movement gained strength but the involvement of feminist groups and activists in different protests, social movements and even political parties has also grown. An example of this was the wide presence of feminist activists in the 15-M movement, many of whom subsequently went into political representation and became part of newly created parties such as Podemos. This participation is both a sign of their commitment to social change and their recognition of the intersectionality of oppression. However, it also makes a complex exercise of reconstruction and defence of the feminist space necessary. Precisely, in this article we set out an analysis of the debates around proposals and strategies held by the feminists of Podemos who, after having participated in social movements, have decided to collaborate with this political platform.

KEYWORDS: Feminisms; political parties; Podemos; political participation; commitment; women in politics. 


\section{INTRODUCCIÓN}

Podemos nace el 17 de enero de 2014 con la presentación en Madrid del manifiesto Mover ficha (https://es.scribd.com/document/199547061/Manifiesto-Mover-Ficha) y con el objetivo de configurar una candidatura para las elecciones al Parlamento Europeo que tendrían lugar pocos meses después. A través del subtítulo del manifiesto, Convertir la indignación en cambio político, Podemos se presenta como un instrumento para canalizar las demandas del movimiento de los indignados o 15-M (Valdivieso, 2017, p. 298). Ante el contexto de crisis económica y de legitimidad institucional, Podemos se ofrece como una alternativa para "hacer llegar a las instituciones la voz y las demandas de esa mayoría social que ya no se reconoce en esta UE ni en un régimen corrupto sin regeneración posible". Así, "aparece como una fuerza outsider" (https://podemos.info/wp-content/ uploads/2016/03/Documento_politico_Podemoscast.pdf), especialmente en comparación con los dos partidos previamente hegemónicos en el tablero político español (con una crítica explícita al bipartidismo del PP-PSOE) (Sola y Rendueles, 2017). “No podemos parecernos a los partidos viejos", se afirma en el Documento Político aprobado en la asamblea estatal de Vistalegre 2 (febrero de 2017). Desde el propio partido se señala, como característica distintiva, la intención de recuperar la soberanía popular, favorecer la participación ciudadana y los procesos participativos (https://files.podemos.info/BL2bQhSWUv.pdf) y, en definitiva, situar a la ciudadanía como eje central en la toma de decisiones.

Por otra parte Podemos, tanto en su documento fundacional como en numerosos textos, propuestas, declaraciones de sus líderes etc., ha venido incluyendo explícitamente la temática de género, apostando por la igualdad entre hombres y mujeres. Muestra de ello es el Documento Organizativo (https://files.podemos.info/9AMI3us6iC.pdf) aprobado en la Asamblea estatal de Vistalegre 2, donde se opta por un "Podemos feminista", en el que no haya ni un solo espacio "en el que no se tenga en cuenta la perspectiva de los feminismos". De hecho, el feminismo fue uno de los protagonistas de la citada Asamblea, en cuya primera jornada las diferentes listas presentaron sus propuestas en materia de igualdad de género. En íntima relación con la existencia de estos debates, propuestas y documentos, también destaca la elevada presencia en Podemos de mujeres con una amplia experiencia de militancia previa en diferentes organizaciones dentro del movimiento feminista.
Ahora bien, ¿en qué se traducen las declaraciones y la alta presencia de mujeres procedentes del activismo feminista? ¿Hasta qué punto es igualitaria la forma en que Podemos se estructura y organiza? ¿Qué rasgos caracterizan el debate que las mujeres feministas de Podemos mantienen dentro del partido? ¿Cómo se relaciona este debate con el existente en el más amplio movimiento feminista en España? Estas preguntas, cuya respuesta constituye el principal objetivo del presente artículo, no han sido abordadas por los ya abundantes estudios sobre Podemos, usualmente centrados en otros aspectos relativos a su estrategia electoral o a sus formas organizativas (Ferrada, 2017; Gerbaudo y Screti, 2017; Gómez y Ramiro, 2019; Kioupkiolis, 2016; MacMiIlan, 2017; Ramiro y Gómez, 2017; Rodríguez, 2016; Rodríguez-Teruel, Barri y Barberà, 2016; Valdivieso, 2017).

A partir fundamentalmente del análisis de documentos generados por los órganos y las mujeres activistas feministas de Podemos (textos redactados para su discusión en Vistalegre 2, materiales de los Encuentros Estatales de Podemos Feminismos, conferencias, artículos de prensa, debates, entrevistas, etc.), se propone dar respuesta a las preguntas planteadas de acuerdo a cuatro ejes de reflexión. En los dos primeros, analizaremos tanto la presencia de mujeres feministas y del propio discurso feminista en Podemos como algunas características del funcionamiento del partido desde una perspectiva de género. Finalmente, en los dos siguientes apartados analizaremos las propuestas en materia de feminismos de las tres corrientes presentes en la Asamblea estatal de Vistalegre $2^{1}$. Así, se analizarán los principales puntos de discrepancia en los debates establecidos: en relación, por un lado, a las preferencias terminológicas (despatriarcalizar versus feminizar el partido) y, por otro, a las distintas estrategias propuestas para revertir las dinámicas internas de su organización.

\section{PRESENCIA FEMINISTA EN PODEMOS}

Afirma Beatriz Gimeno (2014, 17 de noviembre), diputada de Podemos en la Asamblea de Madrid, que "Podemos está lleno de feministas". Estas feministas presentan trayectorias muy variadas, desde aquellas que llevan décadas militando en el feminismo autónomo, en la universidad, en partidos políticos o en sindicatos a feministas jóvenes que, a pesar de tener experiencias previas relativamente cortas, se han implicado activamente en los espacios feministas del partido. Algunas de estas mujeres han pasado a ocupar cargos de responsabilidad dentro de los órganos de Podemos o puestos de representación política a nivel nacional o autonómico. Puede incluso afirmarse, 
siguiendo a Beatriz Gimeno (2014, 17 de noviembre), que el "activismo feminista" de muchas de estas mujeres no "les ha restado posibilidades" a la hora de ser elegidas para los diferentes órganos de dirección y puestos de responsabilidad, "sino al contrario". La presencia y el trabajo de estas mujeres parece haber sido clave en los cambios producidos en el interior del partido en sus pocos años de existencia. Escribe Beatriz Gimeno (2016, 20 de diciembre):

La primera vez que hablé públicamente en un acto de Podemos, el ya mítico acto del cine Palafox [9 de febrero de 2014], nadie habló de feminismo. Los diferentes oradores hablaron de las Mareas que entonces estaban en plena ebullición, pero nadie dijo nada de la Marea Violeta que en esos días estaba en la calle en guerra con el Ministro Gallardón. Una Marea que, además, ganó su guerra contra el ministro.

En los inicios de Podemos, según Sofía Castañón (https://www.youtube.com/watch?v=wBchgcpeJZk), hablar de feminismo en algunos círculos" "era algo que desde luego no comprendían la mayor parte de los [...] compañeros". De hecho, la adopción de un discurso feminista como elemento esencial del partido no fue algo que inicialmente contara con una aceptación unánime. Desde el Documento de Igualdad que presentó la corriente "Recuperar la ilusión" en Vistalegre 2 (Serra, Rodríguez, Moruno, Errejón y Maestre, 2017), se afirma "que en parte nacimos con el lastre de una cierta desconfianza en que algunas temáticas feministas fueran cultural, política y electoralmente ganadoras". Es decir, existía cierto recelo respecto a que el feminismo fuera una estrategia útil para conseguir el objetivo de seducir a la "mayoría social" y crear el partido "transversal" que movilizara al "pueblo" frente a las "élites" (Errejón, 2016, 17 de julio).

Sin embargo, los cambios, ya en los primeros años, han sido muy relevantes, tal y como resume Beatriz Gimeno (2016, 20 de diciembre):

A mí, aquel día del Palafox [...], me sorprendió el olvido del feminismo. Tres años después, en Podemos, esa situación que se dio entonces sería imposible. Ahora todos los sectores del partido hablan de feminismo, todos lo incorporan en sus planteamientos políticos y los tres proyectos principales de entre los que se presentan [en Vistalegre 2] se nombran como feministas en sus reclamos electorales.

En concreto, en el Documento de Igualdad defendido por la lista "Recuperar la Ilusión" en Vistalegre 2 (Serra et al., 2017, pp. 41-42) se destacan algunas de las principales victorias cosechadas por las feministas:
1) la obligatoriedad de incluir áreas de igualdad en todos los consejos ciudadanos; y en el ético, de principios feministas como parte de la identidad de Podemos; 2) la introducción de la economía feminista y de los cuidados; 3 ) la aprobación de las listas cremallera y de órganos paritarios; 4) haber conseguido, con la entrada de Podemos, el Congreso más paritario de la historia de España; y 5) haber instalado el feminismo como eje clave y transversal en Podemos.

Ahora bien, estos cambios parecen responder, en gran medida, al trabajo militante de las mujeres feministas, ya sea desde los círculos o desde el área de igualdad. "Esto es una victoria de las feministas de Podemos. Una victoria duramente trabajada", reclama Beatriz Gimeno (2016, 20 de diciembre). En esta misma línea, Clara Serra denuncia que "el partido ha dejado [...] en los hombros de las mujeres feministas una tarea que tenía que haber sido más compartida por todos" (https://www.youtube.com/watch?v=-MtzwS$\mathrm{F9nXg})^{3}$. Así, fueron las feministas las que tuvieron que "afrontar la [tarea] de convencer acerca de la necesidad, no solamente ética sino también estratégica, de que Podemos apostara por el feminismo como seña de identidad fundamental" (Serra et al., 2017, p. 5).

Por otra parte, a pesar de los logros conseguidos, las feministas de Podemos siguen siendo críticas con su organización. Por ejemplo, Carolina Vaello afirma que Podemos "es un partido que exteriormente no se identifica como partido feminista" y "que interiormente no se está viviendo como tal" (https://www.youtube. com/watch?v=SHM9E-xcKHc). Clara Serra destaca "la falta de transversalidad del feminismo en Podemos"; un feminismo "no siempre apoyado y asumido por toda la organización" (Serra et al. 2017, p. 5). Mientras que Arantxa Rodríguez (https://www.youtube.com/ watch?v=SHM9E-xcKHc) plantea que "el feminismo es una asignatura pendiente" para Podemos. En concreto, se observa un cierto acuerdo a la hora de destacar la distancia existente entre el "impulso feminista" interno, y la imagen que Podemos proyecta hacia el exterior. Beatriz Gimeno (2017, 26 de enero), por su parte, expresa de la siguiente forma esta contradicción:

Es obvio que Podemos tiene un problema con el feminismo. $Y$ es curioso que lo tenga cuando no paramos de hablar de feminismo [...] y cuando, además, en este partido hay más feminismo organizado internamente que en ningún otro. $\mathrm{Y}$, sin embargo, seguimos teniendo un problema con el feminismo. Desde fuera no se ve ese feminismo. No hemos atraído a las académicas, ni las mujeres del movimiento [feminista] cuentan con Podemos, ni Podemos es conocido por la pujanza de su feminismo. 
Podemos estaría proyectando así una imagen de partido masculinizado (aspecto que seguiremos analizando en el siguiente apartado), con una escasa visibilidad de sus figuras y discursos feministas y poco representativo de las realidades de algunas categorías de mujeres. Estas conclusiones parecen coherentes con la mayor brecha de género en términos de voto que presenta Podemos respecto a otros partidos. Así, por ejemplo, según el barómetro del Centro de Investigaciones Sociológicas (CIS) de enero de 2018 la previsión de voto de Unidos Podemos (sin contar con las confluencias que, por otra parte, no muestran tal brecha de género) para unas supuestas elecciones generales era de un $9 \%$ entre los hombres y de un 6,8\% entre las mujeres. Estos resultados son especialmente llamativos si los comparamos con el PSOE, cuyos porcentajes, en ese mismo barómetro, se situaban en el $18,2 \%$ entre los hombres y el $20,1 \%$ entre las mujeres. Este tipo de datos Ileva a la lista "Recuperar la ilusión" (Serra et al., 2017, p. 6) a afirmar que Podemos no ha conseguido convencer "a las mujeres de nuestro país de que [...] es una herramienta útil para ellas".

\section{CRÍTICAS Y ACTIVISMO FRENTE A LAS FORMAS OR- GANIZATIVAS DEL PARTIDO}

Podemos cuenta, en sus órganos directivos, con un importante número de mujeres. De hecho, a excepción de las Secretarías Autonómicas (con solo cuatro mujeres entre las quince Secretarías), el resto de los órganos de dirección respetan el principio de paridad, respondiendo a lo que el partido se impuso en sus propios estatutos (https://files.podemos.info/ XQpfxDwEvT.pdf). Así, por ejemplo, en el Consejo Ciudadano, ocho de las catorce Secretarías están ocupadas por mujeres, con carteras que van más allá de los ámbitos considerados tradicionalmente femeninos. Igualmente, 21 de las 44 Áreas de trabajo están coordinadas por mujeres. Sin embargo, al margen de las cifras y del reparto de responsabilidades, las feministas de Podemos inciden en la falta de visibilidad de las mujeres en el partido, vinculada a un excesivo protagonismo de los líderes masculinos. Un ejemplo significativo de esta visibilidad masculina se puede observar en los carteles que se diseñaron para el día internacional de la mujer del 8 de marzo de 2016. Estos carteles, distribuidos en las redes sociales, muestran a los dos principales líderes de Podemos, Pablo Iglesias e Íñigo Errejón, acompañados del eslogan "Un país con nosotras, ni una menos" (véase "Pablo Iglesias y Errejón, en los carteles de Podemos del Día Internacional de la Mujer Trabajadora"). Se opta así por una comunicación enfocada en las caras masculinas del partido, in- cluso cuando se trata de un evento feminista, como es el día internacional de la mujer. De hecho, desde un inicio, en la estrategia comunicativa de Podemos un elemento clave ha sido la construcción de la figura del líder (Kioupkiolis, 2016), en este caso Pablo Iglesias. Estrategia que ha podido dificultar la configuración y exposición de liderazgos compartidos y de una mayor diversidad de personas.

El segundo aspecto que apuntan las feministas de Podemos cuando se refieren a una organización masculinizada radica en el predominio de una cultura belicista, competitiva y de confrontación en el funcionamiento interno del partido (https://www. youtube.com/watch?v=URqOv5pc4WM). Una cultura que ellas mismas califican de "masculina y machista" (Serra et al., 2017, p. 42), fruto de "la hegemonía de la testosterona" (Mingorance, 2016, 5 de octubre) y coherente con el proyecto inicial de construir una "máquina de guerra electoral" (Serra et al., 2017, p. 42):

[...] prima el enfrentamiento frente al consenso, la agresividad frente al cuidado, y la competitividad frente a la cooperación. La lógica de ganadores y perdedores y de plebiscito constante reafirma esta cultura de la violencia que expulsa sobre todo a las mujeres. Las dinámicas de Podemos son a menudo también muy agresivas y hostiles, tanto en consejos ciudadanos como en círculos, lo cual hace la convivencia muy dura y dificulta la conciliación (Serra et al., 2017, p. 42).

En consecuencia, la participación en Podemos se estaría desarrollando en el marco de unos modos de funcionamiento que privilegian "Ios valores, competencias y prácticas asociados con las formas de masculinidad dominantes" (Shea Baird, 2018, 18 de noviembre). Así, a la presencia de dinámicas "agresivas" (Serra et al., 2017, p. 42), se suma la alta disponibilidad, los amplios horarios, la omnipresencia de las nuevas tecnologías, las reuniones tardías o informales, etc., incompatibles con la conciliación de la participación política y la vida personal. Estas normas implícitas tienen como consecuencia directa la exclusión de las mujeres de los espacios, formales e informales, de toma de decisiones, y el mantenimiento de "redes masculinas de solidaridad, de participación" (https://www.youtube. $\mathrm{com} /$ watch?v=SHM9E-xcKHc). "No queremos que se decidan las cosas en las cañas", denuncia Clara Serra (https://www.youtube.com/watch?v=aAOrDZkA_CY).

En resumen, estas apreciaciones desvelan una estructura androcéntrica, que perjudica a las mujeres y les impide ocupar espacios efectivos de poder. Frente a esta estructura, las militantes feministas constitu- 
yen una voz crítica dentro de su propia formación política. De hecho, la constante presión que ejercen nos lleva a hablar de ellas como un contrapoder dentro de su propia organización, tal y como reconoce Llum Quiñonero (2017, 12 de febrero): “No hemos llegado hasta aquí para ser complacientes; somos feministas, osadas feministas". Las mujeres feministas asumen sin ambages este rol y son conscientes de que los cambios implican inevitables resistencias. De esta forma, reivindican el carácter subversivo del feminismo como movimiento social y político. En palabras de Arantxa Rodríguez: "El feminismo es impugnatorio, es contestatario, es un espacio de conflicto y de resistencia, y nadie abandona privilegios sin batalla" (https://www. youtube.com/watch?v=SHM9E-xcKHc).

\section{EL DEBATE SOBRE LA “FEMINIZACIÓN DE LA POLÍTI- CA": DESPATRIARCALIZAR VERSUS FEMINIZAR}

El 29 de noviembre de 2016, durante un debate organizado por eldiario.es, Pablo Iglesias, a raíz de una pregunta sobre la feminización de la política, contestó lo siguiente:

La feminización no tiene nada que ver con que los partidos políticos tengan más mujeres en cargos de representación, que eso es importante y es necesario [...] La feminización tiene que ver con la forma de construcción de lo político [...] feminizar la política exige construir comunidad en los barrios, en los centros de estudio, sanitarios y de trabajo [...] y cuidar al que tienes al lado (https://www.eldiario.es/politica/reflexion-PabloIglesias-feminizacion-politica_0_585491590.html).

Estas declaraciones, contra las cuales arremetieron miembros del PSOE, como la diputada y militante feminista Ángeles Álvarez o la secretaria general Susana Díaz $^{4}$, y de las que también se hicieron eco otras representantes del movimiento feminista (Requena Aguilar y Borraz, 2016, 29 de noviembre), reabrieron el viejo debate sobre la feminización de la política. Sin embargo, Iglesias en su intervención no hizo sino reproducir parte de las reflexiones que las feministas de Podemos estaban esbozando en los meses previos a la Asamblea de Vistalegre 2. De hecho, en torno a esta asamblea, se generó entre las feministas del partido un intenso debate sobre el feminismo y su relación con el conjunto de la organización. Así, con el objetivo común de construir "un Podemos feminista que haga política feminista hacia dentro y hacia fuera" (Arantxa Rodríguez, https://www. youtube.com/watch?v=SHM9E-xcKHc), se plantearon una serie de discrepancias, no solo en la preferencia por unas determinadas narrativas, como veremos en este apartado, sino también en las estrategias a adoptar.
Para poder situar el debate que se desarrolló en torno a Vistalegre 2, es necesario recordar las tres listas que concurrieron a la Asamblea, ya que son representativas de los diferentes sectores ideológicos dentro del partido:

"Podemos para todas", con feministas como Sofía Castañón e Irene Montero, lista liderada por Pablo Iglesias.

"Recuperar la llusión", con la presencia de Clara Serra y Ángela Rodríguez-Pam, lista liderada por înigo Errejón.

“Podemos en Movimiento", compuesta por la corriente anticapitalista y con especial protagonismo de Beatriz Gimeno e Isabel Serra.

Finalmente, las listas "Podemos para todas" y "Podemos en Movimiento" adoptaron el documento elaborado por el Círculo de Podemos Feminismos, dando lugar a "Feminismo en movimiento para todas". Este documento fue el que finalmente aprobó el conjunto de la Asamblea (véase Podemos. Documentos. Documento de Feminismos). Por su parte, "Recuperar la llusión" defendió, durante todo el proceso asambleario, su propio Documento de Igualdad. Entre los desacuerdos en los planteamientos de ambos bloques destacó la opción por diferentes términos al definir la estrategia a seguir. La lista común "Feminismo en Movimiento para todas" optó por el concepto de despatriarcalizar el partido, mientras la lista "Recuperar la Ilusión" optó por el de feminizar. Bajo estos dos términos que, a primera vista, no parecen tan alejados, se esconden diferentes postulados epistemológicos sobre el sujeto del feminismo, la ética feminista y, de forma más amplia, los objetivos de la lucha feminista.

Estas controversias obviamente no son exclusivas de las feministas de Podemos, sino que reflejan diferentes posiciones dentro de ámbitos de larga tradición en el activismo feminista. De hecho, como planteábamos al principio de este apartado, los debates en el interior de Podemos tuvieron su eco en el exterior del partido, como muestran diversas contribuciones de destacadas activistas y teóricas feministas publicadas por el mismo período en la prensa (Montero, 2016, 3 de diciembre; Posada Kubissa, 2016, 9 de diciembre; Rodríguez Palop, 2016, 30 de noviembre). Se generó así una discusión pública de la que puede destacarse, como primera conclusión, la complejidad del propio concepto de feminización de la política. El significado primario del término, relacionado con promover una mayor presencia de mujeres en las instituciones de representación política y una mayor visibilidad de estas en los puestos de poder, está mayoritariamente asumido como objetivo fundamental (Montero, 2016, 3 de diciembre). Ahora bien, tanto desde las feministas de Podemos como desde fuera de la organi- 
zación, se plantea que esta práctica corre el riesgo de quedarse en lo estético, en las cuotas de paridad, y eso, como apunta Silvia Gil, "no puede constituir el epicentro de nuestra imaginación política" (Serra, Montero, Ferreiro, Rodríguez-Pam y Gil, 2016, 6 de junio). Feminizar la política se convierte, así, en un objetivo más complejo, que implica diferentes dimensiones, y en definitiva "un cambio de paradigma en las formas, contenidos, voces y horizontes" (Ángela Rodríguez-Pam en Serra et al., 2016, 6 de junio). En concreto, en términos de praxis política, implica una voluntad de cambiar las formas de practicarla, incorporando las herramientas del feminismo (Silvia L. Gil en Serra et al., 2016, 6 de junio; Rodríguez Palop, 2019). Así, por ejemplo, Justa Montero (2016, 3 de diciembre) destaca la necesidad de adoptar "formas de hacer política [...] más participativas, más horizontales, más relacionales, frente a las agresivas y competitivas que marca la práctica masculina hegemónica". Sin embargo, algunas de las participantes en estos debates, pese a defender estas nuevas formas de proceder, alertan sobre la necesidad de "desencializar la feminización de la política" (Silvia L. Gil en Serra et al., 2016, 6 de junio). Es decir, plantean la importancia de no considerar determinados valores o formas de hacer política como "propios, naturales de las mujeres", incluyendo, en todo momento "una crítica al carácter construido de su significado social y político" (Montero, 2016, 3 de diciembre).

En el marco de la Asamblea de Vistalegre 2 este debate se cristaliza en torno a la diferenciación de los términos despatriarcalizar versus feminizar. Así, feminizar la política, para las integrantes de la lista "Recuperar la ilusión", tiene como uno de sus ejes fundamentales (obviamente no el único) incrementar la presencia de mujeres en los espacios públicos y en los puestos de liderazgo de la organización. Inciden, así, en la necesidad de copar los espacios de poder dentro del partido: "Cuando decimos feminizar Podemos, implica, desde luego, que haya más mujeres en espacios claves. Creo que hay una falta evidente de mujeres en Podemos, hay que reducir o corregir la hegemonía masculina de Podemos" (Clara Serra en Mingorance, 2016, 5 de octubre). Esta postura es directamente heredera del feminismo de la igualdad, que pone el énfasis en la presencia paritaria de mujeres en la esfera pública. Sin embargo, Beatriz Gimeno, representante de "Feminismo en movimiento para todas", califica esta estrategia de femocracia, al considerar que consiste fundamentalmente en promover la presencia de mujeres en los cargos y en las primeras filas de las instituciones, sin necesariamente apoyarse en las bases militantes. Gimeno lamenta, de esta forma, lo que ella considera una "institucionalización” del feminismo en Podemos:
Recuperar la llusión propone seguir por la senda ya probada en estos tres años. Un feminismo "institucional" [...], vertical, atrincherado en lo orgánico y que no ha tenido capacidad en todo este tiempo de traspasar los muros de Podemos. Un feminismo que es femocracia, cargos feministas, gente trabajando en feminismo pero incapaz de construir desde abajo, de relacionarse con el feminismo organizado, dentro y fuera del partido (Beatriz Gimeno en Borraz, 2017, 26 de enero).

Estas divergencias deben interpretarse a la luz del ADN de Podemos, que es fruto de la unión de tres corrientes: pablista, errejonista y anticapitalista. Las feministas de Podemos ofrecen un buen ejemplo de esta pluralidad ideológica. Clara Serra, por ejemplo, procede del mundo académico y en sus propuestas integra las tendencias más populistas del sector errejonista, mientras que Beatriz Gimeno, con una larga trayectoria en el feminismo autónomo y LGTBQI, defiende los procesos más participativos de los anticapitalistas. De ahí surgen dos concepciones diferentes de la lucha feminista que, en todo caso, comparten gran parte de los objetivos y que en la práctica coinciden al hablar de la necesidad de promover políticas feministas, transversalizar el feminismo en el partido o combatir las lógicas machistas de la organización. En última instancia, desde "Recuperar la ilusión" se defiende el uso de la palabra feminizar por su valor estratégico, ya que se considera más entendible y con mayor capacidad "de interperlar a todo el mundo", "de llegar a las mayorías" (véase Clara Serra - El feminismo en Podemos). Por su parte, el sector anticapitalista prefiere optar por un lenguaje más subversivo, como lo es el término despatriarcalizar, con "una carga de significado mucho más profunda" (Beatriz Gimeno en Feminismo en movimiento y para todas), con "unas connotaciones mucho más políticas" y que evita el riesgo de esencializar determinadas cualidades como supuestamente inherentes a las mujeres (Borraz, 2017, 26 de enero).

\section{LAS CONTROVERSIAS INTERNAS: LA “ÉTICA DE LOS CUIDADOS" Y LA CONCEPCIÓN DEL FEMINISMO}

Independientemente del término que se utilice, la feminización de la política implica colocar en el centro de la agenda cuestiones que eran previamente marginales o consideradas privadas, entre las cuales se sitúa el tema de los cuidados. La noción de cuidados ha sido y sigue siendo objeto de un nutrido debate tanto dentro de los círculos militantes como en la esfera académica (por ejemplo, Carrasco, 2011; Durán, 
2011; Ezquerra, 2011; Gil, 2011). Según Amaia Pérez Orozco (2014, p. 90), en tanto que "actividades residuales a las de mercado", se trata de "aquellas actividades, necesarias para sostener la vida, que se considera más adecuado que se produzcan en círculos de intimidad". Los "círculos de intimidad" a los que se refiere la autora remiten, así, a la invisibilidad de las tareas de cuidado, realizadas principalmente dentro del espacio privado. Estas actividades de cuidado han sido tradicionalmente llevadas a cabo por mujeres que, en su realización, han desarrollado una serie de habilidades y aprendizajes "situados en sus cuerpos, en sus vivencias y en sus experiencias psicosociales" (Rodríguez Palop, 2019, p. 143). Para algunas teóricas y activistas, este savoir faire adquirido constituye un valor fundamental que las mujeres pueden aportar a la "nueva política", tal y como sostiene María Eugenia Rodríguez Palop:

Debemos plantearnos una ética del cuidado en el ámbito de lo público, y me parece que resulta muy liberador en términos sociales y personales pensar en la feminización de la política, desde esta perspectiva, que consiste básicamente en trasladar el aprendizaje de las mujeres y del movimiento feminista afuera. Por esta razón, entre otras, resulta imprescindible tener una experiencia del cuidado (Rodríguez Palop, 2019, p. 143).

Estas teorizaciones son las que Pablo Iglesias intenta recoger en la intervención que referíamos en el apartado anterior, y son retomadas con especial fuerza por parte de las feministas que se integraron en la lista "Recuperar la ilusión". Así, Clara Serra defiende una ética de los cuidados basada en la experiencia femenina, que capacita a las mujeres para transformar las formas y contenidos de la práctica política clásica:

Somos las mujeres las que estamos sobrerrepresentadas en el espacio privado de los cuidados, las que somos más capaces de provocar, con nuestra llegada al espacio público, una transformación de las prioridades políticas. Feminizar la política es traer al debate público y arrojar luz sobre aquellas cuestiones que han permanecido ocultas justamente por haber estado feminizadas, es decir, por haber sido llevadas a cabo por mujeres en la invisibilidad. Feminizar la política es restituir una ausencia que hacía a la política sorda a problemas comunes de todos y de todas (Serra et al., 2016, 6 de junio)

Ahora bien, esta concepción de la feminización de la política enfocada en la ética de los cuidados como ética femenina genera ciertas controversias. En concreto, el principal riesgo que plantea es el de caer en el esencialismo (Montero, 2016, 3 de diciembre). Es decir, en otorgar un supuesto carácter natural a los rasgos, comportamientos y valores tradicionalmente asociados a las mujeres. Tal naturalización ya fue deconstruida por autoras como Simone de Beauvoir, al afirmar que las diferencias entre hombres y mujeres no son biológicas, sino que son el resultado de una construcción social, y caracterizó el debate, desde los años 70, entre el feminismo de la igualdad (que se oponía al mito de una esencia femenina) y el de la diferencia (que buscaba la valoración de una identidad femenina diferenciada de la masculina). En el marco de Podemos, las diferentes corrientes coinciden en destacar el carácter socialmente construido de la ética de los cuidados (Serra et al., 2016, 6 de junio). Sin embargo, son las feministas que conforman la lista "Feminismo en movimiento para todas" las que inciden en la necesidad de evitar cualquier tipo de esencialismo, negando la existencia de "cualidades inherentes a las mujeres" (Beatriz Gimeno en Borraz, 2017, 26 de enero). Isabel Serra, hermana de Clara Serra y figura destacada del sector anticapitalista de Podemos, argumenta en esta línea que:

El término feminizar la política es insuficiente o por lo menos es equívoco, porque parece que decimos que por el hecho de poner mujeres en determinados puestos de dirección o en determinados espacios políticos [...] y poner con ellas [...] esas características, valores femeninos, eso hace que acabemos con las prácticas y con las lógicas machistas y patriarcales (véase Feminismo en movimiento y para todas).

Finalmente, las diversas posturas epistemológicas dentro de Podemos se reflejan en las diferentes concepciones de la lucha feminista. Así, mientras desde "Feminismo en movimiento para todas" se opta por la defensa de un feminismo "colaborativo" (https://www. youtube.com/watch?v=wBchgcpeJZk), que parta de la experiencia participativa del $15 \mathrm{M}$ y de la colaboración con las bases feministas, desde la lista "Recuperar la ilusión" se incide en la necesidad de un "feminismo ganador", que "no se mantenga siempre en la crítica" ni separado del proyecto global del partido (Serra et al, 2016, 6 de junio) $)^{5}$. Se observa, así, una discrepancia de fondo entre las dos listas con respecto a la función del feminismo en las organizaciones políticas. Desde "Feminismo en movimiento para todas", se considera que el feminismo ha de ir necesariamente ligado al trabajo entre mujeres, a la construcción de "un sujeto político feminista", y a la organización de las mujeres "como mujeres y en tanto que mujeres" (véase Feminismo en movimiento y para todas). Por el contrario, 
para las militantes del sector errejonista, el feminismo es, ante todo, transversal, y no se debe construir al margen del proyecto político del partido, tal como lo expresa Clara Serra:

Necesitamos un feminismo comprometido e implicado en el proyecto de cambio general al que pertenecemos si queremos que sea transversal a dicho proyecto, necesitamos feministas implicadas en el proceso y no aisladas en espacios feministas o corremos el riesgo de ser una pieza marginal de este proceso de cambio. Necesitamos un feminismo imbricado y entrelazado con el discurso, la estrategia política, las apuestas y los retos de Podemos (Serra et al., 2016, 6 de junio).

Por su parte, desde "Feminismo en movimiento para todas", se defienden las alianzas entre mujeres, siguiendo las estrategias del movimiento feminista, y consolidando los procesos participativos iniciados con la creación de los Círculos. Según Beatriz Gimeno:

Un partido feminista no lo construyen las áreas, no lo construyen las secretarías ni las responsables, lo construyen las bases feministas [...] empoderadas. Lo construyen las mujeres que se organizan como feministas, los círculos feministas (véase Vistalegre II. Documento de (gualdad).

Apuestan, como plantea Sofía Castañón (https:// www.youtube.com/watch?v=wBchgcpeJZk), por un feminismo construido de abajo a arriba, que tenga una mirada interseccional, y que respalde a las feministas "de arriba", a las que están pugnando en Podemos y en las instituciones. El reto de construir un Podemos feminista pasa, así, por trabajar con las bases y por tejer alianzas con los diferentes colectivos feministas y LGTBQI.

\section{CONCLUSIONES}

El movimiento feminista está logrando aumentar su presencia pública en todos los rincones del planeta, tal y como ha demostrado la iniciativa global \#MeToo, que ha liberado la palabra de las mujeres víctimas de acoso sexual en el ámbito laboral. En España, entre 2013 y 2019 se han producido las mayores movilizaciones de la historia del feminismo español, destacando la marea violeta, la movilización contra el proyecto de la reforma del aborto, la Marcha 7N del año 2015 contra las violencias machistas o las huelgas feministas del 8 de marzo (8M) de 2018 y 2019 . El éxito que han tenido la huelga y las multitudinarias movilizaciones del $8 \mathrm{M}$ confirman el resurgir del activismo feminista y la necesaria atención a sus propuestas en todos los ámbitos de la sociedad. Los partidos políticos no se han mantenido ajenos a este empuje masivo del feminismo.
En concreto Podemos, nacido a raíz de las movilizaciones ciudadanas del $15-\mathrm{M}$, cuenta en sus filas con numerosas mujeres procedentes del movimiento feminista. Estas militantes han visto en el nuevo partido una oportunidad para llevar al centro del tablero político las cuestiones tradicionalmente debatidas por las organizaciones feministas desde la periferia (Serra et al., 2016, 6 de junio). Así, a lo largo de nuestro estudio, hemos observado que, si bien en los inicios de Podemos el feminismo era marginal en la organización, el trabajo constante de las figuras feministas ha cosechado frutos. La incorporación a la agenda del partido de una serie de temáticas que atraviesan el debate feminista actual es hoy en día una realidad, como lo es la presencia significativa de mujeres feministas en los espacios de decisión. Estas mujeres ejercen de contrapoder en una organización que, si bien respalda en teoría el feminismo, en su funcionamiento sigue mostrando dinámicas patriarcales y belicistas. La excesiva masculinización de las caras visibles y la "máquina electoral" en que se convirtió han dificultado la adopción de otras formas organizativas.

Ante ello, las feministas podemistas coinciden en apostar por una transformación de su partido, convirtiéndolo en un modelo de referencia de cara al exterior y en un espacio capaz de acoger la diversidad de todas las mujeres. Sin embargo, las estrategias planteadas para conseguir este objetivo varían en función de las diferentes corrientes ideológicas ( $p a-$ blista, errejonista, anticapitalista) que constituyen el partido: por un lado se postula una mayor conexión con las bases feministas, recuperando la tradición de un feminismo subversivo y disidente; por otro lado se apuesta por una mayor promoción y visibilización de las mujeres feministas dentro de las esferas de poder, siguiendo la línea de un feminismo más institucional. Ahora bien, la construcción de un feminismo popular que logre articular un discurso para todas y todos, y combatir el machismo, constituye el eje central del trabajo político que vienen realizando todas las feministas de Podemos. En este sentido, y retomando las teorizaciones del psicólogo francés Serge Moscovici (1979), podemos afirmar que estas mujeres constituyen una "minoría activa" dentro de su propia cultura política, que tiene la intención de convertirse en una fuente potencial de influencia en las mayorías.

\section{AGRADECIMIENTOS}

Este artículo se ha realizado dentro del marco del Proyecto I+D del Ministerio de Economía y Competitividad titulado Género, Compromiso y Transgresión en España, 1890-2016 (Código: FEM2016-76675-P). 


\section{NOTAS}

[1] Consideramos que el protagonismo que tuvo el debate sobre aspectos claves del feminismo (términos, estrategias, actores) durante esta Asamblea convierte los documentos generados en torno a la misma en material de especial interés para su análisis.

[2] En palabras de Pablo Iglesias (2014, 24 de enero), "Los Círculos Podemos son agrupaciones voluntarias, abiertas y autónomas que hacen suya la Iniciativa Podemos por el cambio político y el protagonismo ciudadano y popular. Hay tantos como grupos de gente dispues- ta a comprometerse en este momento decisivo. Los Círculos pueden ser territoriales o sectoriales".

[3] En este sentido, Clara Serra (2018), en su obra Leonas y zorras. Estrategias políticas feministas insiste en la responsabilidad de los "aliados masculinos" en la transmisión de las reivindicaciones feministas y relaciona "la exclusión del feminismo de los grandes asuntos" y su "olvido en los grandes debates políti$\cos ^{\prime \prime}$ (p. 124) con la marginalidad de los hombres dentro del debate feminista.
[4] "Pablo Iglesias es sexista y reaccionario", denunció Ángeles Álvarez. Por su parte, Susana Díaz declaró: "nuestro país no se puede permitir que la mitad de la inteligencia quede relegada y mucho menos, como decía el señor Iglesias, se dedique a cuidar del otro. Cuidémonos todos unos a otros y dejemos que haya igualdad y paridad en todas las instituciones" (Riveiro y Castro, 2016, 29 de noviembre).

[5] La conceptualización de este "feminismo ganador" es objeto del ensayo publicado por Clara Serra (2018).

\section{BIBLIOGRAFÍA}

Carrasco, C. (2011). La economía del cuidado: planteamiento actual y desafíos pendientes. Revista de Economía Crítica, 11, pp. 205-225.

Durán, M. A. (2011), El trabajo del cuidado en el cuadro macroeconómico. En: Durán, M. A. (ed). El trabajo del cuidado en América latina y España. Madrid, Fundación Carolina, pp. 11-32.

Ezquerra, S. (2011). Crisis de los cuidados y crisis sistémica: la reproducción como pilar de la economía llamada real. Investigaciones Feministas, 2, pp. 175-194. https://doi.org/10.5209/rev_ INFE.2011.v2.38610

Ferrada, R. (2017). The regime's worst nightmare: the mobilization of citizen democracy. A study of Podemos' (aesthetic) populism and the production of affect in political discourse. Cultural Studies, 31 (4), pp. 543-576. https://doi.org/10.108 0/09502386.2016.1264004

Gerbaudo, P. y Screti, F. (2017). Reclaiming Popular Sovereignty: The Vision of the State in the Discourse of Podemos and the Movimento 5 Stelle. Javnost-The Public, 24 (4), pp. 320-335. https://doi. org/10.1080/13183222.2017.1330089

Gil, S. L. (2011). Nuevos feminismos. Sentidos comunes en la dispersión. Madrid: Icaria.

Gómez, R. y Ramiro, L. (2019). The limits of organizational innovation and multispeed membership: Podemos and its new forms of party membership. Party Politics, 25 (4), pp. 534-546. https://doi. org/10.1177/1354068817742844
Kioupkiolis, A. (2016). Podemos: the ambiguous promises of left-wing populism in contemporary Spain. Journal of Political Ideologies, 21 (2), pp. 99-120. https://doi. org/10.1080/13569317.2016.1150136

MacMillan, C. (2017). Welcome to the carnaval? Podemos, populism and Bakhtin's carnivalesque. Journal of Contemporary European Studies, 25 (2), pp. 258-273. https://doi.org/10.1080/1478 2804.2016.1269642

Moscovici. S. (1979). Psychologie des minorités actives. París: Presses Universitaires de France.

Pérez Orozco, A. (2014). Subversión feminista de la economía. Aportes para un debate sobre el conflicto capital-vida. Madrid: Traficantes de Sueños.

Ramiro, L. y Gómez, R. (2017). Radical-Left Populism during the Great Recession: Podemos and Its Competition with the Established Radical Left. Political Studies, 65 (suplemento 1), pp. 108-126. https:// doi.org/10.1177/0032321716647400

Rodríguez, E. (2016). La política en el ocaso de la clase media. El ciclo 15M-Podemos. Madrid: Traficantes de sueños.

Rodríguez Palop, M. E. (2016, 30 de noviembre). Feminizar la política. Eldiario. es [en línea]. [Fecha de consulta: 29 de agosto de 2019]. Disponible en: https:// www.eldiario.es/zonacritica/Feminizarpolitica_6_585901437.htm

Rodríguez Palop M. E. (2019). Revolución feminista y políticas de lo común frente a la extrema derecha. Madrid: Icaria.
Rodríguez-Teruel, J., Barrio, A. y Barberà, O. (2016). Fast and Furious: Podemos' Quest for Power in Multi-level Spain. South European Society and Politics, 21 (4), pp. 561-585. https://doi.org/10.108 0/13608746.2016.1250397

Serra, C. (2018). Leonas y zorras. Estrategias políticas feministas. Madrid: Catarata.

Sola, J. y Rendueles, C. (2017). Podemos, the upheaval of Spanish politics and the challenge of populism. Journal of Contemporary European Studies, 26 (1), pp. 99-116. https://doi.org/10.1080/14782 804.2017.1304899

Valdivieso, J. (2017). The outraged people. Laclau, Mouffe and the Podemos hypothesis. Constellations, 24 (3), pp. 296309. https://doi.org/10.1111/14678675.12287

\section{Recursos en línea}

Borraz, M. (2017, 26 de enero). Así son las propuestas feministas de los sectores de Podemos para Vistalegre 2. Eldiario. es [En línea]. Disponible en: https:// www.eldiario.es/sociedad/propuestasfeminismo-sectores-Podemos-Vistalegre_0_605789605.html

Errejón, I. (2016, 17 de julio). Del asalto al cerco: Podemos en la nueva fase. Eldario. es [En línea]. Disponible en: http://www. eldiario.es/tribunaabierta/asalto-cercoPodemos-nueva-fase_6_538306170.html

Feminismo en movimiento y para todas [vídeo]. [En línea]. Disponible en: https://www. youtube.com/watch?v=iiqw4YUI6o8 
Gimeno, B. (2014, 17 de noviembre). Ante las críticas al feminismo de Podemos [en línea]. Disponible en: https://beatrizgimeno.es/2014/11/17/ante-las-criticas-al-feminismo-de-podemos/

Gimeno, B. (2016, 20 de diciembre). Por un Podemos feminista. El Huffington Post [En línea]. Disponible en: http://www.huffingtonpost.es/beatriz-gimeno/por-un-podemos-feminista_b_13727108.html

Gimeno, B. (2017, 26 de enero). ¿'Feminismo en Podemos? [En línea]. Disponible en: https://beatrizgimeno.es/2017/01/26/ feminismo-en-podemos/

Iglesias Turrión, P. (2014, 24 de enero). Otra vuelta de tuerka. Círculos Podemos. Público [En línea]. Disponible en: http:// blogs.publico.es/pablo-iglesias/760/ circulos-podemos/

La reflexión completa de Pablo Iglesias sobre la feminización de la política. Eldiario.es, 29 de noviembre de 2016. [En línea]. Disponible en: https://www.eldiario.es/ politica/reflexion-Pablo-Iglesias-feminizacion-politica_0_585491590.html

Mingorance, A. (2016, 5 de octubre). Clara Serra: "Hay que corregir la hegemonía de la testosterona en Podemos". Diariocrítico [en línea]. Disponible en: https:// www.diariocritico.com/entrevistas/ clara-serra-podemos

Montero, J. (2016, 3 de diciembre). Feminizar la política. Viento Sur [En línea]. Disponible en: https://www.vientosur. info/spip.php?article11970.

Pablo Iglesias y Errejón, en los carteles de Podemos del Día Internacional de la Mujer Trabajadora. Europa Press. [En línea]. Disponible en: http:// www.europapress.es/nacional/noticia-iglesias-errejon-carteles-podemos-internacional-mujer-trabajadora-20160307185619.html

Podemos. Documentos. Documento de Feminismos. [En línea]. Disponible en: https://files.podemos.info/ET71FDyZ3R.pdf

Podemos. Documentos. Documento Político. [En línea]. Disponible en: https:// files.podemos.info/HihzerN5Ev.pdf

Posada Kubissa, L. (2016, 9 de diciembre). ¿Quién "feminiza la política"? Eldiario. es [En línea]. Disponible en: https:// www.eldiario.es/tribunaabierta/feminiza-politica_6_589051114.html

Presentación de documentos por Clara Serra [vídeo]. [En línea]. Disponible en: https://www.youtube.com/watch?v=MtzwSF9nXg

Quiñonero, L. (2017, 12 de febrero). Las osadas feministas de Podemos. Diario Información [En línea]. Disponible en: http://www.diarioinformacion.com/ opinion/2017/02/12/osadas-feministas/1859836.html

Requena Aguilar, A. y Borraz, M. (2016, 29 de noviembre). Qué es eso de la "feminización de la política”. Eldiario.es [En línea]. Disponible en: https://www. eldiario.es/politica/significa-feminizarpolitica_0_585491697.html

Riveiro, A. y Castro, I. (2016, 29 de noviembre). EI PSOE arremete contra una reflexión de Pablo Iglesias sobre feminiza- ción de la política. Eldiario.es [En línea]. Disponible: https://www.eldiario.es/ politica/PSOE-reflexion-Pablo-Iglesiasfeminizacion_0_585491623.html

Serra, C., Montero, J., Ferreiro, X., Rodríguez-Pam, A. y Gil, S. L. $(2016,6$ de junio). Feminización de la política. La Circular [En línea]. Disponible en: http://lacircular.info/feminizacion-dela-politica/

Serra, C., Rodríguez, Á., Moruno, J., Errejón, I. y Maestre, R. (2017). Recuperar la ilusión. Documento de Igualdad. [En línea]. Disponible en: https://vistalegre2.podemos.info/wp-content/ uploads/2017/02/Recuperarlailusion_ igualdad_def.pdf

Shea Baird, K. (2018, 18 de noviembre). La feminización de la política: Tiros certeros y piedras sobre el tejado. Eldiario.es. Disponible en: https://www.eldiario.es/ tribunaabierta/feminizacion-politicaTiros-certeros-piedras_6_836476362. html

Vistalegre II. Documento de Igualdad [vídeo]. [En línea]. Disponible en: https://www.youtube.com/ watch?v=AxqUiXtAIOg

\section{Otros recursos}

CIS (2018). 32030/Barómetro de enero de 2018. [en línea]. [Fecha de consulta: 7 de marzo de 2018]. Disponible en: http:// www.cis.es/cis/opencm/ES/2 bancodatos/estudios/ver.jsp?estudio=14381 